\title{
PHOTO-RELEASE OF OXYGEN FROM RHODOPSIN SOLUTION
}

\author{
Shigechika FujIsHita \\ Department of Physiology, Osaka Gakugei University, \\ Tennoji-ku, Osaka, Japan
}

In 1964, the author ${ }^{1)}$ reported that rod outer segments isolated from frog retinae performed a non-respiratory oxygen uptake. In another paper $^{21}$ it was reported that rhodopsin solution took up molecular oxygen in the dark, and that the uptake apparently decreased following an illumination with white light (100lux). Such a decrease is conformable to the result reported by HANAWA et al. ${ }^{3)}$ (1955) that the illumination of white light (25 or 100 lux) inhibited respiration of the rod outer segments isolated from toad retinae. Thus, it is very likely that the rhodopsin molecule concerns the inhibition of $\mathrm{O}_{2}$ uptake of rod outer segments following illumination and that the inhibition of the $\mathrm{O}_{2}$ uptake is not identical to the decrease of respiration.

In order to substantiate these points, the present experiments were performed with a solution of rhodopsin extracted from the rod outer segments of frog retinae.

\section{METHODS}

1. Preparation of rhodopsin solution. Frogs (Rana catesbiana) were kept in a dark room for about three hours before use. Their eyes were excised and the rod outer segments were separated from their retinae with KIMURA's floatation technique ${ }^{4)}$. The outer segments thus isolated were put into distilled water and centrifuged again at 10000 r. p. m. for 15 minutes. This procedure was repeated tor three or four times in order to assure that the sucrose used in the isolation procedure was removed completely. For an extraction of rhodopsin, $1 \mathrm{ml}$ of $1 \%$ digitonin solution was added to the collected outer segments and the mixture obtained was kept for 24 hours at $5^{\circ} \mathrm{C}$. The resultant rhodopsin solution was diluted before use with $2 \mathrm{ml}$ of $0.65 \% \mathrm{NaCl}$ solution.

2. Manometric method. The uptake of oxygen $\left(\mathrm{UO}_{2}\right)$ by the rhodopsin solution and a release of oxygen $\left(\mathrm{PRO}_{2}\right)$ from the rhodopsin solution were measured by the Warburg constant volume respiro-meter. The release of oxygen means a negative direction in terms of oxygen uptake changes. All procedures were carried out in a dark room with its temperature kept at $28^{\circ} \mathrm{C}$. The values of $\mathrm{UO}_{2}$ and $\mathrm{PRO}_{2}$ were calculated in reference to the dry weight of rhodopsin-digitonin complex, namely, consumption or

Received for publication April 1, 1966

藤下成周 
release of $\mathrm{O}_{2}(\mu \mathrm{l}) /$ dry weight of rhodopsin-digitonin complex (mg). The dry weight of the rhodopsin-digitonin complex was obtained in the following way; After each mano-. metric experiment, $20 \%$ trichrolic acid was added to the rhodopsin solution, and the denatured rhodopsin was kept for 10 hours at room temperature and was centrifuged. at 3000 r.p. m. for 15 minutes. The precipitate was dried at $100^{\circ} \mathrm{C}$ for 2 hours.

3. Measurement for $p H$ of rhodopsin solution. The $\mathrm{pH}$ value of the rhodopsin solution. was measured by a $\mathrm{pH}$ meter under dim red light at room temperature. In each measurement, $3 \mathrm{ml}$ of rhodopsin solution was prepared in a little vessel $(10 \mathrm{ml} \mathrm{in}$. content) and the $\mathrm{pH}$ value was measured before use.

4. Illumination with monochromatic light. The monochromatic light used for illuminating samples was prepared as follows: White light was projected from a lamp $(150 \mathrm{~W} 100 \mathrm{~V})$ through an interference filter, so that the white light was converted to. monochromatic light. For the purpose of preparing various kinds of monochromatic light, 18 interference filters were used, the wave lengths of the filters being 400, 430, $450,460,470,480,490,500,520,530,540,550,560,580,600,630,660$ and $670 \mathrm{~m} \mu$, respectively.

\section{RESULTS}

1. Effects of illumination upon the $\mathrm{O}_{2}$ uptake of rod outer segments. As described in the previous paper ${ }^{11}$, the rod outer segments isolated from frog retinae took up molecular oxygen at a high rate in the dark, revealing a non-respiratory nature. The $\mathrm{O}_{2}$ uptake was apparently inhibited following illumination (FIG 1).

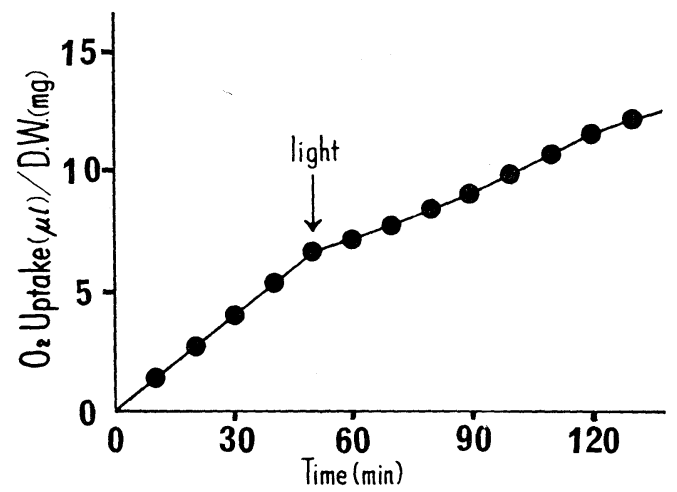

FIG. 1. Inhibitory effect of light on oxygen uptake of isolated rod outer segments.

2. Release of a certain kind of gas following illumination. It was reported ${ }^{2}$, that rhodopsin solution took up molecular oxygen at a high rate in the dark. Upon exposure to light (100 lux), not only an inhibition of $\mathrm{O}_{2}$ uptake, but also a negative change of $\mathrm{O}_{2}$ uptake was observed (FIG 2A). The negative change of $\mathrm{O}_{2}$ uptake may indicate a photo-release of a certain kind of gas from the rhodopsin solution. Occasionally, however, the negative change of $\mathrm{O}_{2}$ uptake following illumination failed to be observed (FIG 2B). In the latter case, it 
was speculated that the negative change might be masked by the $\mathrm{O}_{2}$ uptake of rhodopsin. Therefore, for the purpose of further studying the release of this certain kind of gas, $0.25 \%$ EDTA $^{2)}$ was added to inhibit the $\mathrm{O}_{2}$ uptake of the rhodopsin solution (final concentration of EDTA was $0.025 \%$ ) (FIG 3A). In some experiments, for the purpose of inhibiting the $\mathrm{O}_{2}$ uptake of rhodopsin, the air in a Warburg flask which contained rhodopsin solution was exchanged for nitrogen gas through blowing from a gas bomb (FIG 3B). In both series

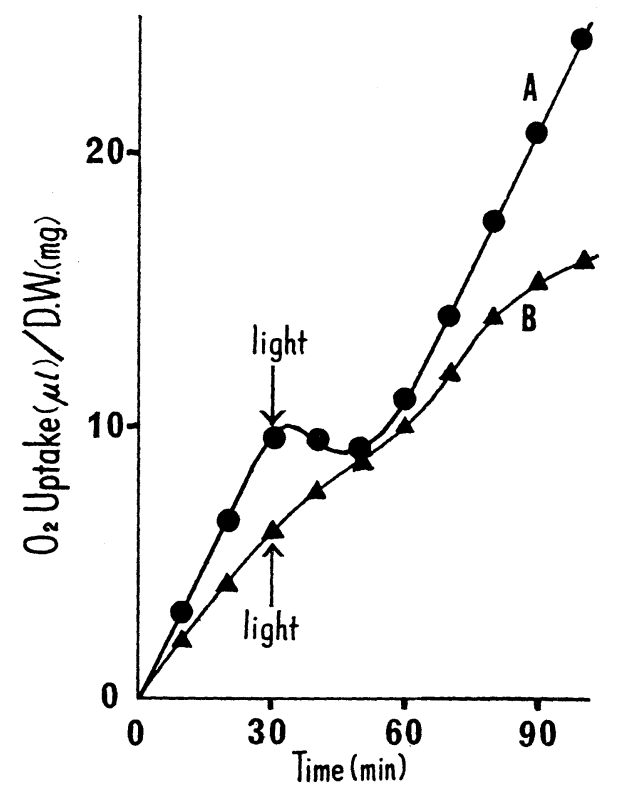

FIG. 2. Inhibitory effect of light on oxygen uptake of rhodopsin solution.

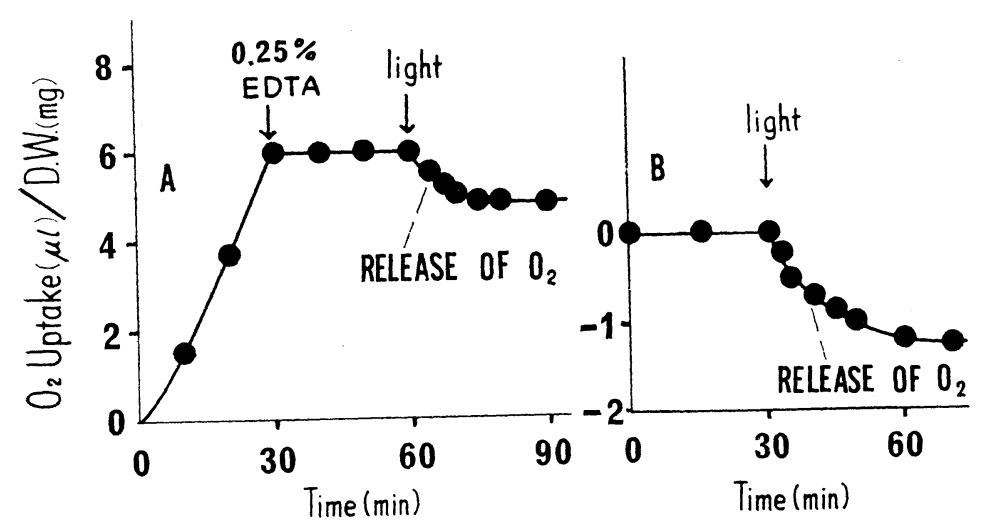

Fig. 3. Photo-release of a certain kind of gas from rhodopsin solution. 
of experiments, release of gas following illumination (100 lux) was observed in a simplified feature (FIG $3 \mathrm{~A}$ and $\mathrm{B}$ ).

3. Photo-release of $\mathrm{O}_{2}$ from the rhodopsin solution. Released gas from rhodopsin following illumination was identified with molecular oxygen as follows: In each of the main chambers of flask $\mathrm{A}$ and $\mathrm{B}, 3 \mathrm{ml}$ of rhodopsin solution $(1 \mathrm{ml}$ of original rhodopsin extracted with $1 \%$ digitonin solution from the outer segments of 30 frogs $+2 \mathrm{ml}$ of $0.65 \% \mathrm{NaCl}$ solution) was prepared. In order to remove $\mathrm{CO}_{2}$ gas from the gas phase in flask $\mathrm{A}, 0.2 \mathrm{ml}$ of $1 \mathrm{~N} \mathrm{KOH}$ solution was put into the center well of flask A (FIG 4). On the other hand, alkaline $10 \%$ pyrogallol (in $1 \mathrm{~N} \mathrm{KOH}$ ) solution in the side arm of flask B was used for removing $\mathrm{O}_{2}$ and $\mathrm{CO}_{2}$ gases from the gas phase in flask $\mathrm{B}$. Glass fibers had previously been put into the side arm of flask $B$, so that the alkaline pyrogallol solution was sucked up by the fibers, to make easier absorption of $\mathrm{O}_{2}$ and $\mathrm{CO}_{2}$ gases. Then the air, which was contained in the gas phase in flask $\mathrm{B}$ and connected manometer, was replaced by nitrogen gas. Following illumination (100 lux) of both flasks, the gas pressure in flask A was increased, but it showed no changes in flask B (FIG 4). An increase of gas pressure in flask A following illumination indicates that the alkaline solution in the center well failed to absorb the released gas. Hence it is clear that the released gas was not $\mathrm{CO}_{2}$. A finding that the gas pressure in flask $\mathrm{B}$ remained constant upon the exposure to light, indicates a release of $\mathrm{O}_{2}$ or $\mathrm{CO}_{2}$ into gas phase. Therefore, it is likely that the released gas was $\mathrm{O}_{2}$.

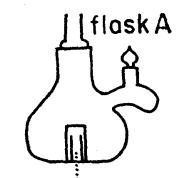

IN $\mathrm{KOH}$

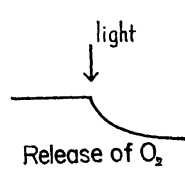

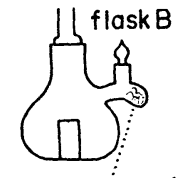

10\% pyrogallol FIG. 4. Discrimination of released gas.

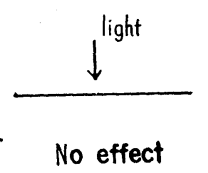

4. Optimum $p H$ for photo-release of molecular oxygen. Two series of experiments were performed for detecting the optimum $\mathrm{pH}$ for a release of molecular oxygen. In the first series of experiments, an oxygen uptake of rhodopsin was inhibited by the addition of $0.25 \% \mathrm{EDTA}^{1,2)}$. In the second series of experiments the air in each flask was replaced by nitrogen gas. In both series of experiments, the optimum $\mathrm{pH}$ for a release of molecular oxygen following illumination was 6.7-6.8 (FIG 5). 


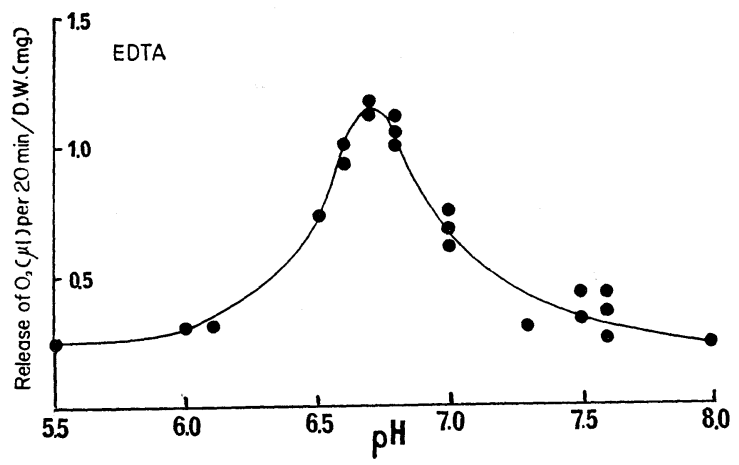

FIG. 5. Optimum pH for photo-release of molecular oxygen from rhodopsin solution.

5. Action spectrum for photo-release of molecular oxygen from rhodopsin solution. If a rhodopsin molecule itself is a pigment which absorbs illuminated light, which causes a release of molecular oxygen, the action spectrum for a release of oxygen from rhodopsin solution should be similar to the absorption spectrum ${ }^{5,6)}$ of rhodopsin, at least partially. Actually, at all wave lengths longer than $450 \mathrm{~m} \mu$, the action spectrum for the release of molecular oxygen shows an agreement with the absorption spectrum of rhodopsin (FIG 6). Below $450 \mathrm{~m} \mu$, however, these spectra are different. Hence it may be concluded that the wave length of efficient light is in a region between $450 \mathrm{~m} \mu$ and

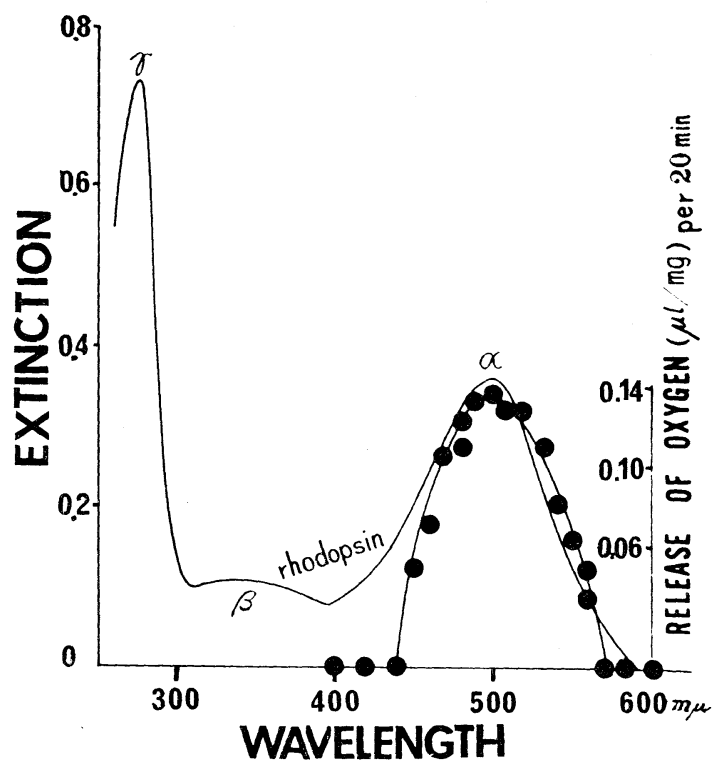

FIG. 6. Absorption spectrum and action spectrum of rhodopsin. 
$560 \mathrm{~m} \mu$, and that the optical energy, absorbed through an interaction between vitamin A aldehyde and opsin, causes a photo-release of oxygen from rhodopsin molecules.

\section{DISCUSSION}

For the purpose of inhibiting the $\mathrm{O}_{2}$ uptake of a rhodopsin solution, $0.25 \%$ EDTA was added to the solution, in some instances, instead of EDTA, the air in the Warburg flask was replaced by nitrogen gas. In both cases, the $\mathrm{O}_{2}$ uptake of rhodopsin solution was perfectly inhibited, so that the photo-release of a certain kind of gas was observed independently of oxygen uptake.

The released gas following illumination was not absorbed by the $\mathrm{KOH}$ solution, so that the gas was not identical with $\mathrm{CO}_{2}$. The released gas was absorbed by alkaline pyrogallol solution, so that it might be either $\mathrm{O}_{2}$ or $\mathrm{CO}_{2}$. Since the released gas was denied to be $\mathrm{CO}_{2}$, it was very likely to be $\mathrm{O}_{2}$.

From the above results, it may be clear that the apparent inhibitory effect of illumination on the $\mathrm{O}_{2}$ uptake of rod outer segments (FIG 1) suspended in $0.65 \% \mathrm{NaCl}$ solution is identical with the photo-release of oxygen from rhodopsin. However, it is able to be assumed that a suspension of rod outer segments took up molecular oxygen by a respiratory pathway, and the respiration of rod outer segments was inhibited following illumination ${ }^{32}$. But, as described in the previous paper ${ }^{1)}$, the non-respiratory nature of oxygen uptake by rod outer segments was experimentally revealed with a denial of the above assumption. Moreover, recently McCoNNELL ${ }^{7)}$ reported that an ascorbateoxidizing system was found in the red fragments of bovine retinal outer segment discs and that the system oxidized ascorbate non-enzymically in the presence of some metal associated with the discs.

The optimum $\mathrm{pH}$ for the photo-release of oxygen was observed when $\mathrm{O}_{2}$ uptake was inhibited with EDTA or $\mathrm{N}_{2}$. In both the cases the optimum $\mathrm{pH}$ was found between 6.7 and 6.8. According to RADDING and WALD ${ }^{8}$ slow changes of $\mathrm{pH}$ of rhodopsin solution following illumination scarcely happened when the initial $\mathrm{pH}$ of rhodopsin solution was between 5.5 and 7 . Outside of this range, they rose nearly symmetrically, and they mainly resulted from acid and alkaline denaturation of opsin. Moreover, WALD and BROWN ${ }^{9)}$ reported that the optimum condition for regeneration of rhodopsin is $\mathrm{pH} 6$, there being little or no regeneration above $\mathrm{pH} 7$ or below $\mathrm{pH} 4.5$. From these reports $^{8,9)}$, it is likely that the most probable physiological $\mathrm{pH}$ range inside of rod outer segments is between 5.5 and 7. Therefore, it is considerable that a photo-release of oxygen from rhodopsin occurs in a physiological $\mathrm{pH}$ range inside of rod outer segments.

According to the first law of photo-chemistry (Grothus-Draper's law), illuminated light should be absorbed by a pigment just before transforming 
the energy from absorbed quanta into chemical energy. Hence, if a rhodopsin molecule concerns with a photo-release of oxygen as a pigment, the action spectrum for the photo-release of oxygen from rhodopsin solution should coincide with the absorption spectrum of rhodopsin. Actually, at all wave lengths longer than $430 \mathrm{~m} \mu$ these two spectra coincide to each other, but below $430 \mathrm{~m} \mu$ they are different (FIG 6).

In the absorption spectrum of rhodopsin, there are three humps named $\alpha, \beta$ and $\gamma$. Hump $\alpha$ (main band) is found between $430 \mathrm{~m} \mu$ and $600 \mathrm{~m} \mu$ and it has $\lambda_{\max }$ at about $500 \mathrm{~m} \mu$, hump $\beta$ (cis band) and hump $\gamma$ (protein band) are found between $310 \mathrm{~m} \mu$ and $400 \mathrm{~m} \mu$ and between $260 \mathrm{~m} \mu$ and $300 \mathrm{~m} \mu$, respectively (FIG 6). Hump $\alpha$ may appear as a result of interaction between opsin and $\pi$-electrons of vitamin A. Hump $\beta$ and $\gamma$ are due to the absorption of light by vitamin A and opsin, respectively. As described above, the action spectrum and absorption spectrum of rhodopsin are similar to each other, while wave length is longer than $430 \mathrm{~m} \mu$. This agreement suggests that the energy of light absorbed by $\pi$-electron bond between vitamin A aldehyde and opsin plays a main role in the photo-release of oxygen from rhodopsin molecule, and that the energy of light absorbed by vitamin A or opsin themselves evoked no effects on the release of oxygen.

Now it is supported to consider that the photo-release of molecular oxygen attributes to a trigger effect of photo-perception in the retina.

\section{SUMMARY}

1. Oxygen uptake of rod outer segments was apparently inhibited following illumination.

2. When rhodopsin solution was illuminated, a photo-release of molecular oxygen from rhodopsin solution was observed, possibly leading to an inhibition of the oxygen uptake of rod outer segments.

3. Optimum $\mathrm{pH}$ for the release of molecular oxygen was 6.7-6.8.

4. Action spectrum for photo-release of molecular oxygen from rhodopsin solution was observed in a spectrum region between $450-560 \mathrm{~m} \mu$.

5. Light absorbed by interaction between $\pi$-electron of vitamin $A$ aldehyde and opsin may contribute to the release of $\mathrm{O}_{2}$ from rhodopsin solution; light absorbed by vitamin $\mathrm{A}$ aldehyde and opsin themselves was non-effective for the release of $\mathrm{O}_{2}$.

The present author wishes to thank Prof. E. KIMURA for his helpful suggestions and criticism in finishing the present study. 


\section{REFERENCES}

1) Fujishita, S. Non-respiratory nature of oxygen uptake by rod outer segments. Jap. J. Physiol. $14:$ 551, 1964.

2) Fujishita, S. Oxygen uptake by rhodopsin solution. Jap. J. Physiol. 16: 61, 1966.

3) Hanawa, I., Kimura, E. And Hosoya, Y. The respiration of the isolated outersegments of rods. Jap. J. Physiol. 5: 322, 1955.

4) Kimura, E. A new method of separating the outersegments of rods from retinal tissue. Jap. J. Physiol. 3: 250, 1952.

5) Collins, F. D. And Morton, R. A. Studies in rhodopsin 3. Rhodopsin and transient orange. Biochem. J. $47: 18,1950$.

6) Collins, F. D., Love, R. M. And Morton, R. A. Studies in rhodopsin 4. Preparation of rhodopsin. Biochem. J. 52: 292, 1952.

7) McConnell, D.G. The isolation of retinal outer segment fragments. J. Cell. Biol. 27 : 459, 1965.

8) RAdDing, C.M. AND WALD, G. Acid-base properties of rhodopsin and opsin. $J$. Gen. Physiol. 39 : 909, 1956.

9) WALD, G. AND Brown, P. K. The role of sulfhydryl groups in the bleaching and synthesis of rhodopsin. J. Gen. Physiol. 35: 797, 1952. 